\title{
Rethinking the Role of the Representativeness Heuristic in Macroeconomics and Finance Theory ${ }^{\dagger}$
}

\author{
Roman Frydman $^{*}$ and Morten Nyboe Tabor**
}

\author{
Working Paper No. 142
}

\author{
December $14^{\text {th }}, 2020$
}

\begin{abstract}
We propose a novel interpretation and formalization of Kahneman and Tversky's findings in the Linda experiment which implies that subjects are rational in the sense of Muth's hypothesis and provides an approach to specifying rational assessment of uncertainty in macroeconomic models. Behavioral-finance theorists have appealed to Kahneman and Tversky's findings as an empirical foundation for a general approach replacing rational expectations. We show that behavioral models' specifications of participants' irrational forecasts and predictable errors are incompatible with Kahneman and Tversky's findings. Our interpretation of Kahneman and Tversky's findings is supportive of Lucas's compelling critique of inconsistent macroeconomic models.
\end{abstract}

\section{https://doi.org/10.36687/inetwp142}

JEL Codes: B41, D80, D81, D91, E71, G41.

Keywords: Uncertainty in Economic Models; Kahneman and Tversky's Experimental Findings; Behavioral Finance; Muth's Hypothesis; REH.

\footnotetext{
${ }^{\dagger}$ The authors are participants in the Program on Knightian Uncertainty Economics at INET. We thank George Akerlof, Søren Johansen and Anders Rahbek for insightful comments and helpful suggestions. We are grateful to INET for support of this research.

${ }^{*}$ Department of Economics, New York University, 19 West 4th Street, New York, NY 10003, USA; e-mail rf3@nyu.edu (Corresponding author)

${ }^{* *}$ Institute for New Economic Thinking (INET)
} 


\section{Introduction}

Daniel Kahneman and Amos Tversky, as well as other experimental psychologists, amassed evidence on how subjects assess uncertainty in a variety of contexts. ${ }^{1}$ Tversky and Kahneman (TK) singled out one finding as being "surprising and less acceptable" (TK, 1983, p. 297). In what has come to be known as the Linda experiment, an overwhelming majority of subjects appeared to have violated the elementary logical rule that the probability of the intersection of uncertain events is smaller than the probability of any of its constituent events.

TK argue that what they interpreted as subjects' "flagrant violation of the conjunction rule" is driven by "seductive... intuitions" (pp. 299, 314). ${ }^{2}$ They called the psychological mechanism underpinning these intuitions the representativeness heuristic.

Behavioral economists have embraced TK's findings as an empirical foundation for their core premise that factors such as judgmental heuristics, framing, intuition, or market sentiment "distort" an individual's assessment of uncertainty. ${ }^{3}$ Importantly, behavioral models rely on a subjective probability measure to represent such psychologically-driven departures from the "objective" probabilities of payoff-relevant outcomes. As a result, a behavioral model presumes that a market participant commits a systematic, predictable error in his assessment of uncertainty. However, TK's findings cannot provide an empirical rationale for modeling this error: Because probabilistic representations of participants' predictable errors necessarily conform to the conjunction rule, they are inherently incompatible with TK's interpretation of their findings, according to which subjects' assessments of uncertainty violate that rule.

What has been largely overlooked is that if subjects' responses really vio-

\footnotetext{
${ }^{1}$ Tversky and Kahneman (1983) provide an extensive review of many experimental findings and further references to the voluminous literature spurred by TK's seminal 1974 article in Science, which, according to Fiedler and von Sydow (2015), "was cited over 7,000 times - an unbelievable rate for a psychology article."

${ }^{2}$ All citations only to page numbers refer to TK (1983).

${ }^{3}$ For a seminal review of "framing effects" and related "biases," see Tversky and Kahneman $(1974,1981)$. For an extensive discussion of behavioral-finance models and further references, see Barberis and Thaler (2003).
} 
lated the conjunction rule in so many settings, TK's findings would be incompatible not only with the behavioral approach, but with much of formal economic theory. Importantly, their findings would be incompatible with models that assume that individuals are "rational," in the sense that their subjective probabilistic assessments of uncertainty about payoff-relevant outcomes are consistent with the "objective" probability of these outcomes. As TK put it,

judgments that [do] not obey the conjunction rule cannot be expected to obey more complicated principles that presuppose this rule, such as Bayesian updating,..., and the maximization of expected utility (p. 313).

However, this far-reaching implication for economic theory hinges on TK's assumption that the representativeness heuristic "blocks" the subjects from recognizing that their responses violate the conjunction rule (p. 300). TK were puzzled about why so many subjects in a wide variety of contexts would make such an obvious error. After all, the simplicity of "compelling logical rules ... makes violations appear implausible" (p. 313).

It is this implausibility that has led us to seek an alternative explanation of subjects' responses. Even if psychological factors influence participants' decision-making, as behavioral economists compellingly argue, incorporating such factors into economic theory would seem to require that market participants adhere to elementary logical rules. Thus, an explanation that presumes that subjects do not make obvious logical mistakes seems crucial for assessing whether TK's findings are relevant for economic theory.

One could of course argue that TK's experimental findings are not relevant for modeling how participants in real-world markets assess uncertainty. In contrast, we follow behavioral economists in assuming that TK's findings do provide an empirical rationale for micro-foundations of macroeconomic and finance theory. However, we propose an alternative interpretation and formalization of subjects' responses that imply that they conform to the conjunction rule and other basic laws of probability. Moreover, our formalization of TK's findings implies that subjects are not only logically coherent but also "ratio- 
nal": their assessments of uncertainty are based on a reasonable understanding of the actual ("objective") uncertainty about the payoff-relevant events.

Our explanation of subjects' responses integrates TK's findings with Muth's (1961) pathbreaking hypothesis that an economist can represent a market participant's rational assessment of uncertainty by specifying a participant's forecasts of market outcomes as being consistent with the economist's own understanding of uncertainty about these outcomes. This has an important, though perhaps surprising, implication: In the context of macroeconomic and finance models, TK's findings provide an empirical foundation for rational expectations models.

Remarkably, TK also pointed to an explanation of their findings that can be integrated with Muth's hypothesis: In assessing uncertainty about events in the Linda experiment, as well as about market outcomes, "representativeness is reducible to similarity" (p. 296). Although TK interpreted representativenessdriven responses as a "surprising ... violation of the conjunction rule," they regarded subjects' assessments of resemblance between uncertain events as "neither surprising nor objectionable" (p. 297).

TK's crucial insight was that subjects' responses in the Linda experiment can be explained in terms of sample statistics, such as frequencies, of the actual "objective" occurrences of relevant uncertain events. Building on this insight, we formalize the concept of resemblance and show how it can explain subjects' responses not as constituting a representativeness-driven "violation of the conjunction rule," as TK proposed, but rather as reflecting a resemblance-based assessment adhering to that rule. This reconciles TK's findings with the seemingly uncontroversial idea that subjects conform to elementary logical rules. Integrating TK's findings with Muth's hypothesis then provides an empirical foundation for modeling rational micro-foundations of macroeconomic and finance models.

As we discuss in Section 10, like the rational expectations hypothesis (REH) or any other representation of an individual's assessments of uncertainty, TK's hypothesis that resemblance-based assessments can be understood in terms of the frequencies of the actual occurrences of uncertain events is necessarily 
boldly abstract. TK neither assumed nor demonstrated empirically that individuals actually estimate such frequencies to assess uncertainty. Indeed, TK (p. 294) emphasize that an individual's assessment of uncertainty could be "deliberate or not," in the sense that he actually collects the data and uses some formal estimation procedure, or relies on his intuition or other informal considerations.

Whereas our resemblance-based explanation of subjects' responses reconciles TK's findings with Muth's model-consistency hypothesis, behavioralfinance economists' attempt to rely on TK's findings as an empirical foundation for their inconsistent intertemporal models suffers from apparently insurmountable problems. A recent book by Gennaioli and Shleifer (GS, 2018) illustrates these difficulties.

Appealing to TK's findings, GS propose what they call diagnostic expectations (DE) as an approach to specifying market participants' assessment of uncertainty in behavioral-finance models. The core premise of $\mathrm{DE}$ is that participants' reliance on the representativeness heuristic "distorts" their assessments of uncertainty, impelling them to commit systematic, predictable errors, relative to the forecasts implied by REH.

GS (pp. 144-152) introduce DE in the context of the Linda experiment, which, owing to the simplicity of the uncertainty confronting the subjects, enabled them to highlight the key features of their approach with no loss of generality (pp. 152-164). Although macroeconomic and finance models are intertemporal and involve uncertainty about continuous outcomes, GS show that DE's application in such models is analogous to its specification of participants' assessment of uncertainty about discrete events in the Linda experiment.

Because TK's findings regarding subjects' responses in the Linda-like experiment have been replicated in many settings, GS (p. 9) argue that, as a general approach to specifying market participants' forecasts, DE could replace REH in building macroeconomic and finance models, as well as in policy analysis. For example, DE assumes that participants' forecast errors move in a quantitatively predictable way with REH-implied forecasts. Relying on such "predictability," GS argue that macroeconomic policy analysis based on DE 
models is not subject to Lucas's (1976) critique, because DE would capture, via participants' supposedly predictable error, how changes in macroeconomic policy would lead participants to revise how they forecast market outcomes. This would enable policymakers to analyze the effects of policy changes on the process driving these outcomes.

The claim that DE could replace REH as a general approach to building behavioral-finance models presumes that DE's specification of participants' assessments of uncertainty is compatible with TK's findings. ${ }^{4}$ We show that even if the representativeness heuristic impels subjects to ignore a basic law of probability, their responses cannot be formalized with DE's probabilistic specification.

To be sure, one could assume, as DE does, that representativeness drives subjects' responses. However, DE's incompatibility with TK's findings suggests that it is an ad hoc specification of market participants' responses, rather than a general approach that could replace REH in macroeconomic and finance theory and policy analysis. Here, our argument formalizes TK's understanding that their findings in the Linda-like experiments do not support the (behavioral) "'truth plus error' model, which assumes a coherent ['objective'] system of beliefs that is perturbed by various sources of distortion and error." Paraphrasing this profound, though largely overlooked, argument in the language of modern macroeconomics and finance theory, TK conjectured that market participants' assessments of uncertainty and forecasts about payoff-relevant outcomes are

not usefully analyzed [with] accurate [REH-implied] precepts and a distorting process [the representativeness heuristic] that produces errors and illusions (p. 313, emphasis added).

\footnotetext{
${ }^{4}$ Gennaioli and Shleifer (2018, pp. 13, 139-140) rely on this point as a reason for proposing representativeness-based DE as a general approach to specifying the micro-foundations of behavioral models. For an early influential example of a behavioral model relying on an ad hoc specification of how market sentiment impels participants to deviate from REH, see Barberis, et al. (1998). It is such specifications that GS have sought to replace with DE. For a recent attempt to do so to explain Shiller's (1981) excess volatility puzzle, see Bordalo, et al. (2020a).
} 
Our argument that DE's model-inconsistent representation is incompatible with TK's findings provides another example of the problems inherent in behavioral economists' premise that market participants commit systematic, predictable errors, and that an economist can specify these errors precisely with a probability measure. In the early 1970s, Lucas compellingly argued that when an economist's representation of an individual's assessment of uncertainty about payoff-relevant events is inconsistent with his own model's representation of this uncertainty, the economist contradicts his own hypothesis that his intertemporal model represents the "objective" uncertainty about these events.

As Lucas (1995, p. 255) pointed out in criticizing adaptive expectations, which underpinned the approaches of the 1960s, macroeconomic and finance models that violate Muth's hypothesis, such as DE and other behavioralfinance models, suffer from a "glaring" inconsistency. ${ }^{5}$ Such models effectively presume that market participants time and again ignore systematic forecast errors, which are predictable according to an economist's model (Lucas, 2005, p. 283).

The paper proceeds as follows. Sections 2 and 3 formally state the relevant events in the Linda experiment, and define representativeness in terms of the frequencies of the occurrences of these events. Section 4 formalizes TK's representativeness-based interpretation of subjects' responses. Section 5 establishes that DE's specification of subjects' supposedly "distorted" assessment of uncertainty is incompatible with TK's findings. Section 6 provides formal support for TK's rejection of the usefulness of the (behavioral) "truth plus error" model for analyzing representativeness-driven assessments of uncertainty. Sections 7- 9 show that subjects' responses interpreted by TK as a violation of the conjunction rule can be understood as reflecting a probabilistic assessment

\footnotetext{
${ }^{5}$ In his Nobel lecture, Lucas (1995, p. 255) recounts how the importance of ridding intertemporal models of such inconsistency persuaded macroeconomists to abandon the micro-founded models of the 1960s and embrace their REH counterparts. For an extensive discussion and formal illustration of this revolutionary development in macroeconomic theory, see Frydman and Goldberg (2007) and Frydman and Phelps (2013).
} 
of resemblance adhering to that rule. Section 10 integrates TK's findings with Muth's hypothesis. We conclude, in Section 11, with a discussion of two companion papers in which the approach we introduce here is applied to extend Lucas's (1978) intertemporal model of asset prices in a way that acknowledges that, like market participants, economists face Knightian uncertainty about this process.

\section{The Linda Experiment}

A classic experiment by Kahneman and Tversky (TK), aiming to examine decision-making under uncertainty, features a fictitious 31-year-old woman, Linda. As a college student, Linda was deeply concerned about discrimination and social justice, and participated in anti-nuclear demonstrations. We refer to a history of engagement in such activities as "progressive," and use $H^{p}$ to denote the set of all individuals with a progressive history.

TK (p. 297) presented the following statements to their experiment's subjects:

- Linda is a bank teller: she is among individuals in set $T$.

- Linda is a bank teller who is also active in the feminist movement (set $F$ ), placing her among the individuals comprising the intersection $T \cap F$.

\subsection{Kahneman and Tversky's Findings}

- When asked about "the degree to which Linda resembles" a feminist bank teller, relative to a generic bank teller (who may be a feminist or not), the subjects ranked $T \cap F$ higher than $T$.

- The subjects were also asked to assess whether it was more or less "probable" that Linda is a feminist bank teller $(T \cap F)$ than that she is generic bank teller $(T)$. An overwhelming majority answered that it was more probable that she is in $T \cap F$ than that she is in $T$ (p. 297). 


\subsection{Formal Design of the Experiment}

Each of the questions in the Linda experiment involves different uncertain events. In order to present formally how TK explain the assessments of uncertainty that underpin a subject's answers to these questions, we consider the population of tellers $(T)$, who are assumed to be college graduates. Among them are those who were progressive while in college $\left(H^{p}\right)$ and those who were not $\left(H^{n p}\right)$. The group of tellers is also partitioned into those who are active feminists $(T \cap F)$ and those who are not $\left(T \cap F^{C}\right)$. This implies that

$$
T=\left(T \cap F \cap H^{p}\right) \cup\left(T \cap F \cap H^{n p}\right) \cup\left(T \cap F^{C} \cap H^{p}\right) \cup\left(T \cap F^{C} \cap H^{n p}\right)
$$

We consider a sample of $n(T)$ bank tellers, which comprises $n(T \cap F)$ feminists and $n\left(T \cap F^{C}\right)$ non-feminists, that is, $n(T)=n(T \cap F)+n\left(T \cap F^{C}\right)$. The sample of tellers includes $n\left(H^{P}\right)$ progressives and $n\left(H^{n p}\right)$ non-progressives, that is, $n(T)=n\left(H^{P}\right)+n\left(H^{n p}\right)$. Moreover, the sample includes information placing a bank teller in one of the intersection of sets partitioning $T$, in (1).

\section{The Representativeness Heuristic}

TK regarded subjects' responses to the first question in the Linda experiment "neither surprising nor objectionable" (p. 297). Building on Tversky (1977) and Gati and Tversky (1982), TK (pp. 296-297) argued that characterizing Linda's history as progressive "improves the match," or resemblance: someone with a progressive history more closely resembles a typical bank teller who is also a feminist than she does a generic bank teller (who may or may not be a feminist).

In contrast, TK considered subjects' responses to the second question "more surprising and less acceptable" (p. 297). They interpreted their subjects' response - that it is more "probable" that Linda, someone in $H^{p}$, is in $T \cap F$ than that she is in $T$ - as an assessment that, conditional on $H^{p}$, the probability of $T \cap F$ is greater than the probability of $T$, that is, $P\left(T \cap F \mid H^{p}\right)>P\left(T \mid H^{p}\right)$. Consequently, TK regarded the overwhelming ma- 
jority of subjects' responses to the second question as "a flagrant violation of the conjunction rule" (p. 299).

TK explained this outcome by arguing that "judgments under uncertainty ... are often mediated by intuitive heuristics that are not bound by the conjunction rule" (p. 293). TK's (p. 294) crucial insight was that a "natural assessment" of uncertainty would be based on the frequency of the actual occurrences of the relevant events. They referred to a "strategy" that relies on such sample summaries as a "judgmental heuristic" (p. 294).

The frequencies necessarily obey the class inclusion rule, that is, the frequency of the intersection of two events is smaller than that of either of its constituents, which, when uncertainty can be represented with a probability measure, implies the conjunction rule. Aiming to explain what they interpreted as subjects' violation of this basic rule of probability, TK (p. 296) defined the heuristic, which they called "representativeness," in terms of the ratio of the relevant frequencies:

Definition 1 "An attribute is representative of a class if it is very diagnostic, that is, if the relative frequency of this attribute is much higher in that class than in a relevant reference class" (p. 296, emphasis added).

In the context of the Linda experiment's second question, "an attribute" of a bank teller is that she is also an active feminist $(T \cap F)$. Definition 1 operationalizes representativeness in terms of the ratio of frequencies of feminist bank tellers $(T \cap F)$ among those individuals who, like Linda, have a progressive history (placing them in a "class," $H^{p}$ ), and those who did not engage in progressive activities while in college (placing them in the complementary "reference class," $\left.H^{n p}\right)$. Denoting these frequencies with $f\left(T \cap F \mid H^{p}\right)$ and $f\left(T \cap F \mid H^{n p}\right)$, respectively, we define the degree of representativeness of $T \cap F$ as follows:

$$
R\left(T \cap F \mid H^{p}, H^{n p}\right)=\frac{f\left(T \cap F \mid H^{p}\right)}{f\left(T \cap F \mid H^{n p}\right)},
$$

where $f\left(T \cap F \mid H^{p}\right)=\frac{n\left(T \cap F \cap H^{p}\right)}{n\left(H^{p}\right)}$ and $f\left(T \cap F \mid H^{n p}\right)=\frac{n\left(T \cap F \cap H^{n p}\right)}{n\left(H^{n p}\right)}$, and $n(A)$ stands for a number of individuals in a set $A$. According to TK's Definition 
$1, T \cap F$ "is representative" of $H^{p}$, relative to $H^{n p}$, if it is "very diagnostic," that is if

$$
R\left(T \cap F \mid H^{p}, H^{n p}\right)>D^{R}>>1,
$$

where $D^{R}$ is some threshold value "much higher" than unity.

Remark 2 Following TK's Definition 1, we define representativeness in terms of frequencies. Because uncertainty is measurable in the situation we consider here, we could have defined representativeness in terms of "objective" probabilities (see, for example, Section 5.2). However, in our companion papers (described in Section 11), we use the approach introduced here to model assessments of Knightian (non-measurable) uncertainty. Thus, we retain TK's definition in terms of the ratio of frequencies, in 11, which makes it applicable regardless of whether uncertainty is measurable or Knightian.

\section{TK's Account of How Representativeness Drives Sub- jects' Asssesments}

TK's explanation of what they interpreted as subjects' violation of an elementary law of probability rests on the idea that a "seductive" psychological mechanism - the representativeness heuristic - drives subjects' answers to the Linda experiment's second question (p. 313). As they put it,

[N]aive subjects generally endorse the conjunction rule in the abstract, but their application of this rule to the Linda problem is blocked by a compelling impression that $[T \cap F]$ is more representative of her than $[T]$ is (p. 300, emphasis added).

TK argued that subjects' assessment that $T \cap F$ is much more representative of Linda than $T$ is, that is, $R\left(T \cap F \mid H^{p}, H^{n p}\right)>>R\left(T \mid H^{p}, H^{n p}\right)$, is triggered by $T \cap F$ being "very diagnostic" of $H^{p}: R\left(T \cap F \mid H^{p}, H^{n p}\right)>D^{R}>>1$. We show that this account of subjects' responses follows, on logical grounds, from TK's definition of representativeness in terms of the ratio of frequencies, in (2). As such, it does not entail the violation of the conjunction rule. TK's 
interpretation that subjects violate the conjunction rule effectively assumes that $R\left(T \cap F \mid H^{p}, H^{n p}\right)>>R\left(T \mid H^{p}, H^{n p}\right)$ constitutes the assessment that $P\left(T \cap F \mid H^{p}\right)>P\left(T \mid H^{p}\right)$. This section presents the steps in this argument.

Proposition 3 Let the events $T \cap F$ and $T \cap F^{C}$ partition bank tellers, $T$, into those who are feminists and those who are not. Analogously to $f\left(T \cap F \mid H^{p}\right)$, in (2), let $f\left(T \cap F^{C} \mid H^{p}\right)$ denote the frequency of $T \cap F^{C}$ among those individuals who have a progressive history $\left(H^{p}\right)$. Using the identity,

$$
f\left(T \mid H^{p}\right)=f\left(T \cap F \mid H^{p}\right)+f\left(T \cap F^{C} \mid H^{p}\right),
$$

we show in Appendix 12 that

$$
R\left(T \cap F \mid H^{p}, H^{n p}\right)>R\left(T \mid H^{p}, H^{n p}\right)
$$

if and only if

$$
R\left(T \cap F \mid H^{p}, H^{n p}\right)>R\left(T \cap F^{C} \mid H^{p}, H^{n p}\right),
$$

where the degrees of representativeness of $T$ and $T \cap F^{C}$ are defined analogously to (2):

$$
R\left(T \mid H^{p}, H^{n p}\right)=\frac{f\left(T \mid H^{p}\right)}{f\left(T \mid H^{n p}\right)} .
$$

and

$$
R\left(T \cap F^{C} \mid H^{p}, H^{n p}\right)=\frac{f\left(T \cap F^{C} \mid H^{p}\right)}{f\left(T \cap F^{C} \mid H^{n p}\right)} .
$$

One would expect the feminist bank tellers $(T \cap F)$ to be more prevalent among the individuals who, like Linda, have a progressive history $\left(H^{p}\right)$, than they are among the individuals without such history $\left(H^{n p}\right)$ :

$$
R\left(T \cap F \mid H^{p}, H^{n p}\right)=\frac{f\left(T \cap F \mid H^{p}\right)}{f\left(T \cap F \mid H^{n p}\right)}>1 .
$$

In contrast, one would expect $T \cap F^{C}$ to be more prevalent among $H^{n p}$, than they are among $H^{p}$ : 


$$
R\left(T \cap F^{C} \mid H^{p}, H^{n p}\right)=\frac{f\left(T \cap F^{C} \mid H^{p}\right)}{f\left(T \cap F^{C} \mid H^{n p}\right)}<1 .
$$

This proposition reveals the channel through which representativeness drives a subject's assessments that " $[T \cap F]$ is more representative of [Linda] than $[T]$ is." However, instead of appealing to "a compelling impression," as TK do, the proposition shows that this assessment reflects "deliberate[ly] or not" the logical implication of TK's definition of representativeness.

TK's Definition 1 refers to $T \cap F$ as representative if it is very diagnostic, in (3). The following corollary to Proposition 3 clarifies the role that a very diagnostic $T \cap F$ plays in TK's representativeness-based account of what they interpret as subjects' "violation of the conjunction rule."

Corollary 4 Using the identity $f\left(T \mid H^{p}\right)=f\left(T \cap F \mid H^{p}\right)+f\left(T \cap F^{C} \mid H^{p}\right)$ in $R\left(T \mid H^{p}, H^{n p}\right)$, in (7), we show in Appendix 12 that

$R\left(T \cap F \mid H^{p}, H^{n p}\right)-R\left(T \mid H^{p}, H^{n p}\right)=\lambda\left[R\left(T \cap F \mid H^{p}, H^{n p}\right)-R\left(T \cap F^{C} \mid H^{p}, H^{n p}\right)\right]$,

where $0<\lambda<1$, represents the impact of the extent of representativeness of $T \cap F$ relative to $T \cap F^{C}$ on the representativeness of $T \cap F$ relative to $T$.

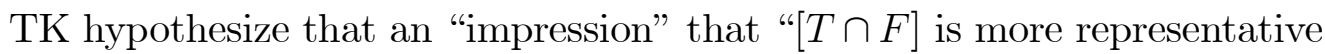
of [Linda] than $[T]$ is" becomes "compelling" when $T \cap F$ is very diagnostic. Corollary 4 shows that, when $R\left(T \cap F \mid H^{p}, H^{n p}\right)>D^{R}>>1$, the difference $R\left(T \cap F \mid H^{p}, H^{n p}\right)-R\left(T \cap F^{C} \mid H^{p}, H^{n p}\right)$ becomes sufficiently large (via (11)) to render $R\left(T \cap F \mid H^{p}, H^{n p}\right)>>R\left(T \mid H^{p}, H^{n p}\right)$.

\subsection{From Representativeness to "Probableness"}

Proposition 3 and Corollary 4 highlight the role played by TK's definition of the degree of representativeness in terms of the ratio of frequencies, in (7)(9), in their account of subjects' responses. However, the implication that, when $R\left(T \cap F \mid H^{p}, H^{n p}\right)>D^{R}>>1$, the inequality, $R\left(T \cap F \mid H^{p}, H^{n p}\right)>>$ $R\left(T \mid H^{p}, H^{n p}\right)$, can account for subjects' belief that it is more "probable" that 
Linda is in $T \cap F$ than that she is in $T$ requires explicitly relating a subject's response regarding how "probable" an event is to the event's representativeness. We define such a relationship with what we call the probableness function, which we calibrate by formalizing TK's interpretation of their empirical findings.

Definition 5 Let $r_{A}=R\left(A \mid H^{p}, H^{n p}\right)$ and $r_{B}=R\left(B \mid H^{p}, H^{n p}\right)$ denote the degrees of representativeness of events $A$ and $B$, respectively, where $H^{p}$, and $H^{n p}$ denote "a class" and its complement. Noting that $r_{A} \in(0, \infty)$ and $r_{B} \in$ $(0, \infty)$, let $\pi(r)$ denote a real-valued function from $[0, \infty)$ to $[0,1]$, which we constrain to be monotonically increasing in representativeness, $r$, that is,

$$
\text { If } r_{1}>r_{2} \text {, then } \pi\left(r_{1}\right)>\pi\left(r_{2}\right) \text {. }
$$

Kahneman and Tversky (and other experimental psychologists) replicated their finding - that subjects' assess the conjunction as more "probable" than one of its constituents - in many Linda-like experiments and in a variety of contexts. Although these experiments differ in a number of specific details, they share one key feature: by design, they ask the subjects to "consider cases where the ordering of events according to representativeness appears obvious":

An event $A$ is judged more probable than an event $B$ whenever $A$ appears more representative than $B$ (Kahneman and Tversky, 1982, p. 33, emphasis added).

We formalize TK's experimental design - that $T \cap F$ is "obviously" more representative than $T$ - with $R\left(T \cap F \mid H^{p}, H^{n p}\right)>>R\left(T \mid H^{p}, H^{n p}\right)$. Letting $A=T \cap F$ and $B=T$ in Definition 5, we formalize TK's account of their findings that subjects' responses regarding the relative probableness of the two events reflect the "obvious" ordering of the events' representativeness:

$$
\text { If } r_{T \cap F}>>r_{T} \text {, then } \pi\left(r_{T \cap F}\right)>\pi\left(r_{T}\right) \text {, }
$$

where $r_{T \cap F}$ and $r_{T}$ denote $r_{T \cap F}=R\left(T \cap F \mid H^{p}, H^{n p}\right)$ and $r_{T}=R\left(T \mid H^{p}, H^{n p}\right)$, respectively. 


\subsection{Formalizing TK's Account of Their Findings with Probableness}

TK hypothesize that subjects' reliance on the representativeness heuristic can explain their belief that it is more "probable" that Linda, someone in $H^{p}$, is in $T \cap F$ than that she is in $T$. We state this formally with a corollary,

Corollary 6 Suppose that Proposition 3 and Corollary 4 hold. Then, when $r_{T \cap F}>D^{R}>>1$,

$$
r_{T \cap F}>>r_{T},
$$

which, from TK's account of their empirical findings, in (13), implies that

$$
\pi\left(r_{T \cap F}\right)>\pi\left(r_{T}\right) .
$$

\subsubsection{Probableness versus Probability}

Proposition 3 and Corollary 4, and thus $R\left(T \cap F \mid H^{p}, H^{n p}\right)>>R\left(T \mid H^{p}, H^{n p}\right)$ are premised on the class inclusion rule that $f\left(T \cap F \mid H^{p}\right)<f\left(T \mid H^{p}\right)$ and $f(T \cap$ $\left.F \mid H^{n p}\right)<f\left(T \mid H^{n p}\right)$. Via Corollary 6, $R\left(T \cap F \mid H^{p}, H^{n p}\right)>>R\left(T \mid H^{p}, H^{n p}\right)$ explains subjects' responses that it is more "probable" that Linda, someone in $H^{p}$, is in $T \cap F$ than that she is in $T$. Thus, ranking $T \cap F$ relative to $T$ by their representativeness as well as by their "probableness" conforms to the basic laws of probability. Consequently, TK's interpretation of their findings as constituting "a flagrant violation of the conjunction rule" requires an additional key assumption:

Assumption $\mathbf{7}$ The subjects' responses that it is more "probable" that Linda, someone in $H^{p}$, is in $T \cap F$ than that she is in $T, \pi\left(r_{T \cap F}\right)>\pi\left(r_{T}\right)$, express their assessment that $P\left(T \cap F \mid H^{p}\right)>P\left(T \mid H^{p}\right)$.

This assumption formalizes TK's (p. 314) assertion that "a seductive" psychological mechanism - the representativeness heuristic - "blocks" the subjects from recognizing that they are violating the conjunction rule. 


\section{Behavioral-Finance Models' Incompatibility with TK's Findings in the Linda Experiment}

Our formalization of TK's interpretation of subjects' responses to the experiment's second question underscores the key distinction between an assessment of the probability of an event, $P\left(T \cap F \mid H^{p}\right)$, and a representativeness-driven assessment of the event's probableness, $\pi\left(r_{T \cap F}\right)$. Whereas probability necessarily conforms to the conjunction rule, $P\left(T \cap F \mid H^{p}\right)<P\left(T \mid H^{p}\right)$, representativeness, and thus probableness, by design are not bound by that rule: when $r_{T \cap F}>D^{R}>>1, \pi\left(r_{T \cap F}\right)>\pi\left(r_{T}\right)$.

Thus, even if a very diagnostic $T \cap F$ impels a subject to violate the conjunction rule, as TK have argued, a subject's assessment of uncertainty could not be formalized with a subjective probability measure, which necessarily obeys that rule. Behavioral-finance theorists have had to sidestep this seemingly uncontroversial point in order to embrace TK's findings as an empirical rationale for the probabilistic micro-foundations of their models.

\subsection{TK's Findings and the Specification of Behavioral- Finance Models}

A behavioral-finance model represents the actual ("objective") uncertainty about payoff-relevant outcomes as well as an individual's subjective assessment of that uncertainty with probability measures.

\subsection{1 "Objective" Probability}

In the Linda experiment, the "objective" uncertainty about the relevant events, such as $T \cap F$ in a class $H^{p}$, takes a particularly simple form: whether an individual who engaged in progressive activities while in college, in $H^{p}$, is or is not currently (at the time of the experiment) a feminist bank teller, in $T \cap$ $F$. A behavioral model would represent this uncertainty with the conditional probability, $P\left(T \cap F \mid H^{p}\right)$, which we define as follows:

Definition 8 Let $f\left(T \cap F \mid H^{p}\right)=\frac{n\left(T \cap F \cap H^{p}\right)}{n\left(H^{p}\right)}$ denote the frequency of actual occurrences of the event, $T \cap F$ in a class $H^{p}$. Then the conditional probability, 
$P\left(T \cap F \mid H^{p}\right)$ is defined, if

$$
\begin{gathered}
\lim _{n\left(H^{p}\right) \rightarrow \infty} f\left(T \cap F \mid H^{p}\right)=\lim _{n\left(H^{p}\right) \rightarrow \infty} \frac{n\left(T \cap F \cap H^{p}\right)}{n\left(H^{p}\right)}=p_{\left(T \cap F \mid H^{p}\right)} \text { as } n(T \cap F) \rightarrow \infty, \\
\text { where } 0 \leq p_{\left(T \cap F \mid H^{p}\right)} \leq 1 \text { is a finite constant. }
\end{gathered}
$$

Because $f\left(T \cap F \mid H^{p}\right)$ is the frequency of the actual occurrences of the event $T \cap F$ in a class $H^{p}$, we refer to $p_{\left(T \cap F \mid H^{p}\right)}$ as the objective probability of $T \cap F$, conditional on $H^{p}{ }^{6}{ }^{6}$

\subsubsection{Representing a Rational Assessment of Measurable Uncer- tainty}

As we discuss in Section 12, Muth (1961, p.316) advanced the pathbreaking hypothesis that an economist can acknowledge that a market participant is rational (reasonable) by specifying his forecasts of market outcomes as being consistent with the economist's own understanding of uncertainty about these outcomes. Applying Muth's hypothesis in models that assume that uncertainty about outcomes is measurable has led economists to characterize a participant's "rational" assessment of this uncertainty with the "objective" probability measure implied by the model. We state this formally, in the context of the Linda experiment as follows:

Definition 9 Suppose that an economist has reasons to hypothesize that the actual (objective) uncertainty about the event, $T \cap F$ in a class $H^{p}$, is measurable, and thus that it can be represented with the conditional probability, $P\left(T \cap F \mid H^{p}\right)$. We refer to the (probability-conforming) subjective assessment, $P^{S}\left(T \cap F \mid H^{p}\right)$, as rational, if $P^{S}\left(T \cap F \mid H^{p}\right)=P\left(T \cap F \mid H^{p}\right)$.

An overwhelming majority of economic models rely on probabilistic rationality to represent an individual's assessment. For example, a typical macro-

\footnotetext{
${ }^{6}$ For an extensive discussion of the frequentist definition of probablity that we rely on here and a statement of Von Mises' (1957) axioms ensuring that the limit in (14) converges to a probability measure, see Interpretations of Probability (Stanford Encyclopedia of Philosophy, 2019) and references therein.
} 
economics and finance model hypothesizes that, at any point in time, uncertainty about payoff-relevant events at all future times can be characterized with a probability measure, conditional on information available to market participants. According to Definition 9, this measure represents a participant's rational understanding of uncertainty about future market outcomes. The conditional expectation of this probability is typically referred to as the rational expectation (REH) representation of an individual's forecast.

\subsection{3 "Distorted" Subjective Probability}

A behavioral model represents psychologically-driven departures from the "objective" probabilities of payoff-relevant outcomes, such as $P\left(T \cap F \mid H^{p}\right)$, with a "distorted" subjective probability measure, which we denote with $P^{S}(T \cap$ $\left.F \mid H^{p}\right)$. As a result, a behavioral model's account of a market participant's assessment of uncertainty presumes that he commits a systematic error, $e(T \cap$ $\left.F \mid H^{p}\right)=P\left(T \cap F \mid H^{p}\right)-P^{S}\left(T \cap F \mid H^{p}\right)$, that is observable and predictable on the basis of the information available to him: $\left(H^{p}\right)$.

Lacking a unified approach to specify "distorted" subjective probabilistic assessments, $P^{S}\left(T \cap F \mid H^{p}\right)$, and thus to identify such errors, early generations of behavioral models formalized them with myriad context-specific insights. ${ }^{7}$ Although many of these insights seem relevant for understanding how individuals cope with uncertainty, early behavioral models amounted, as Thaler (2017, pp. 489-490) put it in his Nobel lecture, to a collection of interesting "stories" rather than "a research paradigm."

Kahneman and Tversky (and other experimental psychologists) replicated their finding that subjects assess the conjunction as more "probable" than one of its constituents in many Linda-like experiments. For Thaler, relying on the representativeness heuristic to formalize such an error in individual assessments would turn the hodgepodge of early behavioral models into "something resembling [a] science... [of] predictable errors" in how individuals make assessments under uncertainty (emphasis in the original).

However, Thaler seems to have overlooked that TK's findings cannot pro-

\footnotetext{
${ }^{7}$ See Gennaioli and Shleifer (2018, pp. 13, 139-140) for an extensive discussion of this important point.
} 
vide a representativeness-based rationale for individuals' supposedly "predictable errors," and thus for behavioral models' "distorted" probabilistic assessments of uncertainty. Even if representativeness drives subjective assessments, and a very diagnostic $T \cap F$ impels subjects to violate the conjunction rule, such assessments cannot be formalized with a probability measure, which necessarily obeys that rule.

\subsection{Diagnostic Expectations}

A recent book by Gennaioli and Shleifer (GS, 2018) illustrates the insuperable obstacles standing in the way of any attempt to build a behavioral-finance model on the basis of TK's findings. To do so, GS introduce diagnostic expectations (DE) in the context of the Linda experiment.

Because they assume that uncertainty faced by individuals is measurable, GS (2018, p. 145) replace the frequencies in Kahneman and Tversky's definition of representativeness, in (2), with the respective objective probability measures:

$$
R^{D E}\left(T \cap F \mid H^{p}, H^{n p}\right)=\frac{P\left(T \cap F \mid H^{p}\right)}{P\left(T \cap F \mid H^{n p}\right)},
$$

GS formalize the "distorted" assessments of uncertainty as follows:

$$
P^{D E}\left(T \cap F \mid H^{p}\right)=P\left(T \cap F \mid H^{p}\right)\left[R^{D E}\left(T \cap F \mid H^{p}, H^{n p}\right)\right]^{\theta} c_{1},
$$

where $P^{D E}\left(T \cap F \mid H^{p}\right)$ represents a subjective DE assessment of uncertainty, $\theta>0$ formalizes the degree of "distortion," and $c_{1}$ is one of the constants ensuring that the joint distribution, $P^{D E}\left(F, T, H^{p}, H^{n p}\right)$, which underpins the conditional probability $P^{D E}\left(T \cap F \mid H^{p}\right)$, is defined. Analogously, GS represent a subject's assessment of the uncertainty of the event, $T$ in a class $H^{p}$, with a probability measure, $P^{D E}\left(T \mid H^{p}\right)$.

\subsubsection{DE's "Predictable" Errors}

Hypothesizing that the actual uncertainty about the event, $T \cap F$ in a class $H^{p}$, as well as a subjective assessment of this uncertainty, can be represented 
with probability measures, $P\left(T \cap F \mid H^{p}\right)$ and $P^{D E}\left(T \cap F \mid H^{p}\right)$, respectively, presumes that market participants commit a systematic, predictable error, $e^{D E}\left(T \cap F \mid H^{p}, H^{n p}\right)$, which can also be represented with a probability measure:

$$
\begin{aligned}
e^{D E}\left(T \cap F \mid H^{p}, H^{n p}\right) & =P^{D E}\left(T \cap F \mid H^{p}\right)-P\left(T \cap F \mid H^{p}\right) \\
& =\left\{\left[R^{D E}\left(T \cap F \mid H^{p}, H^{n p}\right)\right]^{\theta} c_{1}-1\right\} P\left(T \cap F \mid H^{p}\right)(17)
\end{aligned}
$$

DE implies that whenever $T \cap F$ is representative of $H^{p}$, that is, $R^{D E}(T \cap$ $\left.F \mid H^{p}, H^{n p}\right)=\frac{P\left(T \cap F \mid H^{p}\right)}{P\left(T \cap F \mid H^{n p}\right)}>1$, a subject overestimates $T \cap F$ 's "objective" uncertainty, $P^{D E}\left(T \cap F \mid H^{p}\right)>P\left(T \cap F \mid H^{p}\right)$, commits the "error," $e^{D E}(T \cap$ $F \mid H^{p}, H^{n p}$ ), which predictably depends on the information that the experiment, by design, makes available to him: that Linda has a progressive history $\left(H^{p}\right)$.

\subsubsection{Do TK's Findings Provide an Empirical Rationale for Diag- nostic Expectations in Behavioral-Finance Models?}

Building on their specification in the context of the Linda experiment, GS (2018, pp. 152-191) develop diagnostic expectations in an intertemporal finance model. They argue that, because TK's findings have been replicated in many contexts, DE provides a unified approach to specifying market participants' assessments of uncertainty in behavioral-finance models. As Bordalo, et al. (2020b) asserted, DE is a "psychologically founded ...model of belief formation" (p. 2749, emphasis added).

To be sure, TK did find that in many Linda-like experiments, a majority of subjects responded that it is more "probable" that someone like Linda (in $\left.H^{p}\right)$ is in a conjunction $(T \cap F)$ than that she is in one of its constituents $(T)$. However, even if one embraces TK's interpretation of such responses as "a flagrant violation of the conjunction rule," as behavioral economists have done, the supposedly "distorting" influence of the representativeness heuristic on subjects' assessments cannot be formalized with DE or any other probability measure.

As we formally showed in Section 4, TK's representativeness-based interpretation of subjects' responses presumes that, when the conjunction is very 
diagnostic - that is, $R^{D E}\left(T \cap F \mid H^{p}, H^{n p}\right)>D^{R}>>1$ - subjects violate the conjunction rule: They erroneously believe that, conditional on information available to them $\left(H^{p}\right)$, the probability of the conjunction is greater than the probability of one of its constituents. In contrast, DE, in (16), necessarily satisfies the conjunction rule, that is, $P^{D E}\left(T \cap F \mid H^{p}\right)<P^{D E}\left(T \mid H^{p}\right)$, regardless of whether $T \cap F$ is very diagnostic or not.

Our analysis in this section casts doubt on any attempt to develop an approach to building macroeconomic and finance models, or to economic theory more broadly, that aims to explain outcomes as the result of predictable errors in an individual's representativeness-driven assessments. Remarkably, TK argued that their findings did not support a behavioral "truth plus error" model. Moreover, they questioned the usefulness of a research strategy that sought to understand outcomes as driven by such systematic, predictable errors:

perception is not usefully analyzed into a normal ["objective"] process that produces accurate precepts $\left[P\left(T \cap F \mid H^{p}\right)\right]$ and a distorting process that produces errors $\left[e^{D E}\left(T \cap F \mid H^{p}, H^{n p}\right)=P^{S}(T \cap\right.$ $\left.F \mid H^{p}\right)-P\left(T \cap F \mid H^{p}\right)$, in (17)] and illusions (p. 313, emphasis added).

\section{Representativeness-Driven Assessments Do Not Pre- dictably Overestimate or Underestimate "Objective" Probabilities}

Behavioral "truth plus error" models assume that, owing to their reliance on the representativeness heuristic or the influence of other psychological factors, market participants commit systematic errors. Moreover, these errors are predictable, in the sense that an economist can characterize them with a probability measure, conditional on the information available to participants, according to his model. For example, DE always overestimates the probability of a representative event, that is, $P^{D E}\left(T \cap F \mid H^{p}\right)>P\left(T \cap F \mid H^{p}\right)$, in (17), if $R\left(T \cap F \mid H^{p}, H^{n p}\right)>1$. 
Like DE, TK's account of subjects' responses rests on the premise that the representativeness heuristic drives subjects' assessments of uncertainty about payoff-relevant events away from these events' "objective" probability. However, once we replace DE's probabilistic specification with the representativenessbased non-probabilistic formalization of probableness, the timing and magnitude of subjects' overestimation and underestimation crucially depend on psychological factors that appear to be inherently unpredictable. Developing this argument provides formal support for TK's assertion that subjects' representativeness-driven responses are not "usefully analyzed with a 'truth plus error" model.

Our argument builds on our formalization in Section 4.1 of TK's hypothesis that subjects' assessments of how "probable" an event is can be understood in terms of the event's representativeness. To this end, we formulate the following specification of the probableness function, in Definition 5:

$$
\pi(r)=\pi_{\delta}(r)=1-\delta^{r},
$$

where $\delta \in(0,1)$, which implies that $\pi(r)$ maps $r \in[0, \infty)$ onto $[0,1]$ monotonically, that is,

$$
\text { If } r_{1}>r_{2} \text {, then } \pi\left(r_{1}\right)>\pi\left(r_{2}\right) \text {. }
$$

The parameter $\delta$ formalizes TK's idea that the "impression" that representativeness makes on subjects' assessments about how "probable" an event is could be "sticky." As subjects' perception becomes stickier, in the sense that the value of $\delta$ increases (see graph), the effect of changes in representativeness on their assessment of probableness becomes more attenuated. 
Figure 1

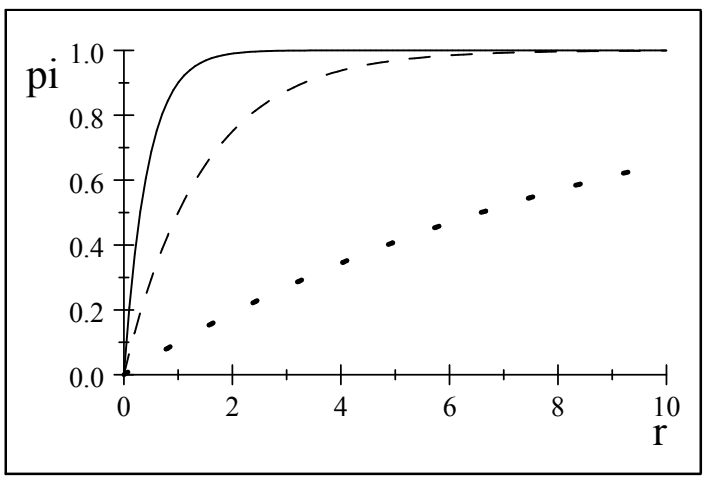

Solid: $\delta=0.1$; Dash: $\delta=0.5$;

Dots: $\delta=0.9$.

\subsection{The Unpredictability of "Errors" Engendered by the Representativeness Heuristic}

The parametric specification of the probableness function in (18) implies a subject relying on the representativeness heuristic to assess measurable uncertainty, $P\left(T \cap F \mid H^{p}\right)$, would commit the following error:

$$
e^{R}\left(r_{T \cap F}\right)=1-\delta^{r_{T \cap F}}-P\left(T \cap F \mid H^{p}\right)
$$

This, in turn, implies that

$$
e^{R}\left(r_{T \cap F}\right)<0, \text { if } 1<r_{T \cap F}<\frac{\log \left(1-P\left(T \cap F \mid H^{p}\right)\right)}{\log (\delta)},
$$

which shows that a subject's assessment of the probableness, $\pi\left(r_{T \cap F}\right)$, of a representative event, $r_{T \cap F}>1$, could either overestimate or underestimate the event's "objective" probability, $P\left(T \cap F \mid H^{p}\right)$. For example, as $r_{T \cap F} \rightarrow \infty$, the difference in (20) would eventually turn positive. However, as $\delta \rightarrow 1$ - that is, as a subject's representativeness-based assessment of probableness becomes "stickier" - the condition in (21) implies that $e^{R}\left(r_{T \cap F}\right)$ in (20) could be negative even when $T \cap F$ is very diagnostic, that is, $r_{T \cap F}>D^{R}>>1$. 
The condition in (21) also highlights the inherent difficulty of predicting whether and when representativeness would impel a subject to overestimate or underestimate the "objective" probability. Such a prediction depends on the relative values of the degree of representativeness, $r_{T \cap F}$, and the parameter $\delta$, which formalizes how a subject's assessment of uncertainty about $T \cap F$ depends on $T \cap F$ 's representativeness.

Predicting whether market participants' reliance on the representativeness heuristic would lead them to overestimate or underestimate the "objective" probability of outcomes becomes even more problematic in the context of macroeconomic and finance models. This is precisely what Fama (1998) finds in a largely overlooked study of the temporal stability of supposedly systematic behavioral "anomalies" in movements of asset returns: "apparent overreaction to information is about as common as underreaction" (p. 283). To predict when overestimation or underestimation would occur requires specifying ex ante when and how the "psychological effect" of representativenes, which is formalized here with the parameter $\delta$ in (18), is likely to change.

Moreover, there is a voluminous literature documenting that the parameters of the "objective" probability of outcomes undergo structural breaks (see Perron, 2009, for an authoritative review). Such breaks imply that $r_{T \cap F}$ and $P\left(T \cap F \mid H^{p}\right)$ change over time, at least intermittently. According to inequality in $(21)$, whether overestimation or underestimation occurs depends on the relative magnitudes of $\delta, r_{T \cap F}$ and $P\left(T \cap F \mid H^{p}\right)$. Thus, even if $\delta$ is time-invariant, which is a rather strong condition, the change in $r_{T \cap F}$ and $P\left(T \cap F \mid H^{p}\right)$ further compounds the difficulties inherent in specifying macroeconomic and finance models that could predict the timing and magnitude of overestimation or underestimation engendered by participants' supposed reliance on the representativeness heuristic. ${ }^{8}$

Behavioral-finance economists have had to assume away what seem to be insuperable obstacles to formalizing TK's representativeness-based interpre-

\footnotetext{
${ }^{8}$ For recent evidence that psychological considerations, such as extrapolation of past trends or market sentiment, influence participants' forecasts of stock returns at times and in ways that would be difficult to represent with a probability measure, see Frydman and Stillwagon (2018) and Frydman, et al. (2020).
} 
tation of their findings with a probabilistic model. For example, in a recent paper, Bordalo, et al. (2020b, p. 2749) asserted that DE's probabilistic specifications of how representativeness drives market participants' assessments can explain systematic and predictable "overreaction" of participants' expectations relative to those implied by an REH-implied ("objective") probability measure.

In the foregoing section, we showed that supposedly "predictable errors," implied by DE's probabilistic specification of participants' representativenessdriven assessments of uncertainty, are inconsistent with TK's findings. Consequently, we relied in this section on our non-probabilistic formalization of these findings with a model of probableness, which, by design, reflects the event's representativeness. We showed that the heuristic would impel the subjects' to either overestimate or underestimate the event's "objective" probability. Moreover, whether overestimation or underestimation would occur depends on factors that would be inherently difficult to predict with a formal model. These implications of TK's findings show that, as TK (p.13) surmised, "the truth plus error model" does not provide a useful approach to specifying empirically relevant models of how representativeness drives individuals' assessments of uncertainty in either Linda-like experiments or in real-world markets.

\section{Reconciling Kahneman and Tversky's Findings with Formal Economic Theory}

TK's interpretation of their subjects' responses - that it is more "probable" that Linda is in $T \cap F$ than that she is in $T, \pi\left(r_{T \cap F}\right)>\pi\left(r_{T}\right)$ - as constituting an assessment that $P\left(T \cap F \mid H^{p}\right)>P\left(T \mid H^{p}\right)$ presumes that subjects ignore the fact that every feminist bank teller with a progressive history is also a bank teller with that history. TK's (p. 300) explanation why the subjects commit such an obvious error rests on Assumption 7: "a compelling impression" that $T \cap F$ is much more representative of Linda than $T$ is, $R\left(T \cap F \mid H^{p}, H^{n p}\right)>>$ $R\left(T \mid H^{p}, H^{n p}\right)$ "blocks" the subjects from recognizing that they are violating the conjunction rule.

We have shown that DE's formalization of subjects' supposedly "predictable 
errors" triggered by the representativeness heuristic is inconsistent with TK's interpretation of how the heuristic drives subjects' assessments of uncertainty. Moreover, TK's interpretation is incompatible with much of economic theory, which assumes that individuals are "rational." This core premise requires, at a minimum, that individuals adhere to compelling logical rules. (see Section $10)$.

Recognizing that subjects in the Linda experiments are rational in this minimal sense requires an alternative explanation of TK's findings. We propose that a subject's response to the second question in the Linda experiment be understood in the same way as his response to the first question. According to our proposed explanation, both responses are an assessment that Linda, someone in $H^{p}$, resembles a feminist bank teller $(T \cap F)$ more closely than a generic bank teller $(T)$.

Our resemblance-based explanation of a subject's response - that it is more probable that Linda is among feminist bank tellers $(T \cap F)$ than that she is among tellers $(T)$ - necessarily satisfies the class inclusion rule. Thus, interpreting what TK regarded as a representativeness-driven "violation of the conjunction rule" as a resemblance-based assessment adhering to that rule enables us to reconcile TK's findings with the seemingly uncontroversial idea that subjects' judgments conform to the basic rules of logic.

\subsection{Representativeness as Resemblance}

Although TK defined how the representativeness heuristic drives subjects' responses to the Linda experiment's second question in terms of the ratio of frequencies, they clearly thought of the heuristic's much broader applicability as a "natural strategy" for assessing "correspondence" between uncertain events. As they put it,

Representativeness is an assessment of the degree of correspondence... between [an attribute and a class], an act and an actor, a sample and a population or, more generally, between an outcome and a model (pp. 295-296). 
TK (p. 296) argued that "when the model and the outcome are described in the same terms "representativeness is reducible to similarity" (p. 296). TK single out two particular correspondences as examples of how representativeness can be understood as resemblance between two uncertain events:

1. "Because a sample and a population... can be described by the same attributes (e.g. central tendency and variability), the sample appears representative if its salient statistics match [resemble] the corresponding parameters of the population."

2. "A person [Linda] seems representative of a social group [bank tellers who are also feminists, $T \cap F$ ] if her personality [having a progressive history, $H^{p}$ ] resembles the stereotypical member of that group." ${ }^{9}$.

Resemblance-based assessments of the correspondence between a sample and a population play a key role in our approach, which relies on TK's findings as an empirical rationale for specifications of a rational market participant's assessment of uncertainty in macroeconomic and finance models. Formalizing the closer resemblance of Linda, someone in $H^{p}$, to a feminist bank teller $(T \cap F)$ than to a generic bank teller $(T)$, provides a simple way to illustrate how TK's findings can be reconciled with economic theory's core premise that market participants are rational, in the sense of Definition 9 (which formalizes Muth's hypothesis, in Section 10, in the context of the Linda experiment).

\section{Operationalizing Resemblance-Based Assessments}

TK's crucial insight was that subjects' assessment of uncertainty can be understood in terms of the frequencies of the actual occurrences of the relevant events. In Sections 3 and 4, we showed how TK explained subjects' supposed "violation of the conjunction rule" in terms of subjects' reliance on representativeness, which TK formally defined with the ratio of frequencies, such as $R\left(T \cap F \mid H^{p}, H^{n p}\right)=\frac{f\left(T \cap F \mid H^{p}\right)}{f\left(T \cap F \mid H^{n p}\right)}$

\footnotetext{
${ }^{9}$ However, "representativeness is not always reducible to similarity ... A particular act (e.g., suicide) is representative of a person because we attribute to the actor a disposition to commit the act, not because the act resembles the person" (p. 296).
} 
In contrast, TK did not provide a formal definition of "resemblance" that could explain subjects' assessment that Linda, someone with a progressive history, more closely resembles a typical bank teller who is also a feminist than she does a generic bank teller. We provide an explanation of these responses in terms of the frequencies of actual occurrences of three events: $H^{P}$ in a class $T \cap F, H^{P}$ in a class $T \cap F^{C}$, and $H^{P}$ in a class $T$. We implement TK's insight by formalizing subjects' resemblance-based assessments in terms of the frequencies of these events: $f\left(H^{P} \mid T \cap F\right), f\left(H^{P} \mid T \cap F^{C}\right)$, and $f\left(H^{P} \mid T\right)$, respectively.

\subsection{The Key Role of Information about Linda's Pro- gressive History}

One would expect that 31-year old women who work as bank tellers would be less prevalent among those who are active feminists $(T \cap F)$, relative to their peers who are tellers who are not $\left(T \cap F^{C}\right)$. Thus, describing Linda as "merely a 31-year old woman" would not make her appear different from a generic bank teller $(T)$.

However, based on Tversky's (1977) and Gati and Tversky's (1982) experimental evidence, TK (pp. 296-297) argued that adding Linda's progressive history to her description substantially enhances her resemblance to a bank teller who is also a feminist, relative to a generic bank teller (who may or may not be a feminist). We implement TK's insight to formalize an assessment of resemblance triggered by the information about Linda's history.

One would expect that the individuals with a progressive history, $\left(H^{p}\right)$, are more prevalent among feminist bank tellers than they are among the tellers who are not active feminists, that is

$$
f\left(H^{p} \mid T \cap F\right)>f\left(H^{p} \mid T \cap F^{C}\right),
$$

where $f\left(H^{p} \mid T \cap F\right)=\frac{n\left(H^{p} \cap T \cap F\right)}{n(T \cap F)}$ and $f\left(H^{p} \mid T \cap F^{C}\right)=\frac{n\left(H^{p} \cap T \cap F^{C}\right)}{n\left(T \cap F^{C}\right)}$.

Applying TK's insight that subjects' assessments of uncertainty can be explained in terms of such frequencies, we formalize subjects' responses regarding 
the degree of resemblance of Linda, someone in $H^{p}$, to $T \cap F, T \cap F^{C}$, and $T$ with $f\left(T \cap F \mid H^{P}\right), f\left(T \cap F^{C} \mid H^{P}\right)$, and $f\left(T \mid H^{P}\right)$, respectively.

The inequality in (??) operationalizes an assessment that Linda more closely resembles $T \cap F$ that she does $T \cap F^{C}$. The following proposition shows that (22) implies that the individuals with a progressive history, $\left(H^{P}\right)$, are more prevalent among $T \cap F$ than they are among $T$ :

Proposition 10 We show in Appendix 12 that

$$
f\left(H^{p} \mid T \cap F\right)>f\left(H^{p} \mid T \cap F^{C}\right)
$$

implies that

$$
f\left(H^{p} \mid T \cap F\right)>f\left(H^{p} \mid T\right) .
$$

This proposition formalizes TK's finding that subjects' responses reflect their assessment that Linda resembles a feminist bank teller more closely than resembles a generic bank teller. Moreover, because the inequality in (23) does not entail the violation of the class inclusion rule, our formalization of resemblancebased assessments of uncertainty is consistent with TK's assertion that subjects' responses to the experiment's first question are "neither surprising nor objectionable."

\section{Resemblance-Based Assessments as Estimation of Prob- abilities}

The preceding section formalized the resemblance-based explanation of a subject's assessments of uncertainty about events in the Linda experiment in terms of the frequencies of the events' occurrences. Hypothesizing that uncertainty about the experiment's events is measurable, these frequencies are the maximum likelihood estimates of the events' probabilities. 


\subsection{A Probabilistic Interpretation of Subjects' Responses to the Experiment's First Question}

For example, for the sample of occurrences of the event $H^{p}$ in the class $T \cap F$, the frequency, $f\left(H^{p} \mid T \cap F\right)=\frac{n\left(H^{p} \cap T \cap F\right)}{n(T \cap F)}$, is the standard maximum likelihood estimator of $P\left(H^{p} \mid T \cap F\right)$. This shows that, when uncertainty is measurable, our explanation of subjects' responses to the first question of the Linda experiment has a straightforward probabilistic interpretation, which we state as a corollary to Proposition 10:

Corollary 11 Suppose that the uncertainty about the events in the Linda experiment is measurable and that

$$
f\left(H^{p} \mid T \cap F\right)>f\left(H^{p} \mid T \cap F^{C}\right) .
$$

Then, Proposition 10 implies that a subject's assessment that $H^{p}$ resembles $T \cap F$ more closely than it resembles $T$ can be understood as his assessment that $P\left(H^{p} \mid T \cap F\right)>P\left(H^{p} \mid T\right)$, in the sense that the maximum likelihood estimates of these probabilities, $\widehat{p}_{H^{p} \mid T \cap F}$ and $\widehat{p}_{H^{p} \mid T}$, respectively, satisfy the following inequality,

$$
\widehat{p}_{H^{p} \mid T \cap F}=f\left(H^{p} \mid T \cap F\right)>\widehat{p}_{H^{p} \mid T}=f\left(H^{p} \mid T\right) .
$$

This corollary shows that TK's idea - that "the sample appears representative [population] if its salient statistics resemble the corresponding parameters of the population" (p. 296) - can be formalized as an estimation problem.

\subsection{Understanding the "Violation of the Conjunction Rule" as a Probability-Conforming Resemblance- Based Assessment}

TK interpreted a subject's answer to the experiment's second question as reflecting his assessment that $P\left(T \cap F \mid H^{p}\right)>P\left(T \mid H^{p}\right)$. However, the words "likely" and "probable," as well as "likelihood" and "probability," are typi- 
cally defined as synonyms, and dictionary definitions of these words reflect ordinary usage, such as by the subjects in the Linda-like experiments. Consequently, when uncertainty is measurable, a subject's responses - that it is more "probable" that Linda, someone in $H^{p}$, is in $T \cap F$ than that she is in $T$ can be understood as his assessment that the probability that $H^{p}$ is in $T \cap F$ is greater than the probability that she is in $T$, that is, $P\left(H^{p} \mid T \cap F\right)>P\left(H^{p} \mid T\right)$.

Recognizing, as TK pointed out, that in the context of the Linda experiment "representativeness is reducible to similarity," we propose that a subject's response to the experiment's second question in the Linda experiment be understood in the same way as his response to the first question. According to our proposed explanation, both responses are an assessment that Linda, someone in $H^{p}$, resembles a feminist bank teller $(T \cap F)$ more closely than a generic bank teller $(T)$. Thus, neither of the responses entails "violation of the conjunction rule."

\section{Reframing Muth's Hypothesis}

Whereas the representativeness-driven interpretation of TK's findings implies that subjects violate the conjunction rule when the conjunction is very diagnostic, our resemblance-based explanation of subjects' responses to both questions in the Linda experiment is compatible with the minimal threshold of rationality - that subjects always conform to elementary logical rules. However, although logical coherence is "the touchstone of human rationality" (p. 313), much more is required for an assessment to be considered "rational": it must be based on a reasonable understanding of the actual ("objective") uncertainty about the payoff-relevant events.

According to Webster's Dictionary, the words "reasonable" and "rational" are close synonyms. Although economic models' operationalization of "rational" decision-making is more specific, it shares an important feature with its natural-language usage: both tie rationality closely to a reasonable understanding of the world in pursuit of goal-oriented behavior. As Webster's defines it, "rational usually implies a latent or active power to make logical inferences and draw conclusions that enable one to understand the world about 
him and relate such knowledge to the attainment of ends."

Arguing that economists should acknowledge market participants' rationality, Muth appealed directly to the rationale for formal economic models: A model represents an economist's theoretically- and empirically-based understanding of the actual ("objective") uncertainty about the relevant events. Consequently, Muth (1961, p.316) advanced a pathbreaking hypothesis, which we state as follows.

(Muth's) Hypothesis 12 An economist can acknowledge that a market participant is rational (reasonable), in the dictionary sense, by specifying a participant's forecasts of market outcomes as being consistent with the economist's own understanding of uncertainty about these outcomes, as formalized by his model. ${ }^{10}$

Muth implemented his hypothesis in a model that represented the uncertainty about payoff-relevant events as measurable. It was this implementation that came to be known as the rational expectations hypothesis. However, Muth's hypothesis can be applied more broadly. It requires only that the representation of an individual's assessment be consistent with the specification of the actual uncertainty about outcomes, as formalized by an economist's model. ${ }^{11}$ We refer to this broader applicability of Muth's hypothesis 12 as the neo-Muthian hypothesis, which we state as follows.

(The Neo-Muthian) Hypothesis 13 An economist can acknowledge that a market participant is rational by specifying a participant's assessment of uncertainty and his forecasts as being consistent with

1. his own model's characterization of uncertainty about payoff-relevant outcomes, or

\footnotetext{
${ }^{10}$ Contrasting his argument with Simon's (1959) so-called bounded rationality hypothesis, Muth (1961, p.316) emphasized that his model-conistency hypothesis reflects "exactly the opposite point of view: that dynamic economic models [of the 1950s] do not assume enough rationality" on the part of market participants.

${ }^{11}$ For an early argument that Muth's hypothesis may be applied so broadly, see Frydman and Goldberg (2015).
} 
2. an adequate approximation of his model, estimated on the basis of a sample of realizations and its variables.

When uncertainty about events in the Linda experiment is measurable, defining an adequate approximation of this uncertainty is straightforward. For example, the frequency, $f\left(H^{p} \mid T \cap F\right)$, provides an adequate approximation of the probability, $P\left(H^{p} \mid T \cap F\right)$, in the sense that this frequency converges asymptotically to the true value of this probability.

TK's insight was that sample statistics, such as $f\left(H^{p} \mid T \cap F\right)$, can explain an individual's resemblance-based assessments of uncertainty about the event $H^{p}$ in the class $T \cap F$. Invoking the neo-Muthian hypothesis, $f\left(H^{p} \mid T \cap F\right)$ therefore represents a subject's rational assessment of uncertainty about this event.

In reframing Muth's hypothesis to entail consistency between an economist's representation of a market participant's assessment of uncertainty about payoff-relevant outcomes and the adequate approximation of his model's specification of this uncertainty, we do not assume that market participants actually rely on the estimation procedures. Like all other formal representations of an individual's assessments of uncertainty, representing resemblance-based assessments as an estimation based on past data about uncertain outcomes is necessarily boldly abstract.

Even if, as in behavioral models, formal representations of individuals' forecasts are based on empirical evidence, they are not literal portrayals of how market participants assess the uncertainty that they face or of how they forecast outcomes. As Muth emphasized, his model-consistency hypothesis "does not assert that the scratch work of entrepreneurs resembles the system of equations in any way; nor does it state that predictions of entrepreneurs are perfect or that their expectations are all the same" (Muth, 1961, 317, emphasis in original). This is why Muth's hypothesis is so central to building macroeconomic and finance models. Because economists cannot literally portray the diverse ways in which market participants assess uncertainty, the hypothesis offers a tractable, "sensible" way to acknowledge that these participants' assessments are related to a presumably rational - albeit imperfect - understanding of the 
process driving outcomes, as formalized by an economist's own model.

\section{Concluding Remarks}

We follow Gennaioli and Shleifer in assuming that TK's findings provide an empirical foundation for macroeconomics and finance models. However, we have shown that these findings are incompatible with diagnostic expectations' core premise that the representativeness heuristic impels the subjects to commit systematic, predictable forecast errors, relative to the "objective" probability of uncertain events. In contrast to DE's formalization, our resemblance-based explanation of TK's findings implies that subjects' assessments of uncertainty are consistent with the "objective" probability of uncertain events, as specified by an economist's model.

Whereas the design of the Linda experiment suggests that the "objective" uncertainty about the experiment's events is measurable, in a nonexperimental setting, the uncertainty may be measurable or not. ${ }^{12}$

We have developed our resemblance-based explanation of TK's findings and reframed Muth's hypothesis with a view toward using it to build consistent macroeconomics and finance models that acknowledge that economists, like market participants, face Knightian uncertainty. According to Knight, uncertainty about the consequences of economic decisions is often, at least in part, non-repetitive (novel). As he put it,

Business decisions... deal with situations which are far too unique ... [to rely solely on] statistical tabulations. The conception of objectively measurable probability is simply inapplicable (Knight, 1921, pp. 231-232, emphasis added).

By definition, Knightian uncertainty cannot "by any method" - even with the benefit of hindsight (on the basis of past data), let alone ex ante - "be reduced

\footnotetext{
${ }^{12}$ One could design a Linda-like experiment in which the uncertainty about whether someone who has a progressive history is among feminist bank tellers depends on the historical events since they graduated from college. Because historical events are at least in part non-repetitive, such a design would render uncertainty about the experiment's events nonmeasurable, or Knightian, in the sense defined below.
} 
to an objectively measurable probability" (Knight, 1921, p. 231, 321). Such non-measureable uncertainty, Knight (p. 198) argued, arises from changes affecting payoff-relevant events that occur at times and in ways that cannot be represented with probabilistic rules, such as Markov switching. ${ }^{13}$

When outcomes undergo such unforeseeable change, the sample of their observations comes, at least intermittently, from different probability measures. Herein lies the key importance of TK's insight that a market participant's assessment of uncertainty can be understood in terms of sample statistics of observations of the process characterizing outcomes: A model's approximation based on such samples is defined, regardless of whether uncertainty about the outcomes is measurable or Knightian.

Recognizing that uncertainty about market outcomes cannot be represented with REH models is increasingly viewed as crucial to remedying the shortcomings of macroeconomic and finance theory. For example, in his Nobel lecture, Hansen (2013, p. 399, emphasis added) argues that REH models "miss something essential: uncertainty [arising from] ambiguity about which is the correct model."

By integrating TK's finding and the neo-Muthian hypothesis 13 the approach introduced here enables economists to acknowledge that one of the main reasons why market participants and economists face ambiguity about the process driving outcomes is that this process undergoes unforeseeable change. Moreover, because the neo-Muthian hypothesis represents market participants' forecasts as being consistent with an economic model's specification of Knightian ("objective") uncertainty about outcomes, our approach enables an economist to build macroeconomic models that are not subject to the Lucas critique.

Lucas (1976) showed that as the "objective" uncertainty about market outcomes undergoes change, for example due to shifts in macroeconomic policy, participants' would revise how they forecast outcomes. Assuming that the "objective" uncertainty about market outcomes is measurable, economists have

\footnotetext{
${ }^{13}$ For a review of theoretical developments and references to the large literature representations of change with the Markov chain models, see Hamilton (2008).
} 
relied on REH to analyze precisely how changes in policy might affect market outcomes. This implication of REH models has persuaded central banks and other policymaking institutions to rely on them to analyze consequences of alternative macroeconomic policies.

To acknowledge that market outcomes are subject to unforeseeable change, that rational market participants understand this, and that policymakers thus face Knightian uncertainty is to recognize the inherent pitfalls of relying on REH models in policy analysis. Applying the neo-Muthian hypothesis to represent participants' forecasts as being consistent with an economic model's approximation under Knightian uncertainty does enable an economist to analyze how policy changes might influence revisions of participants' forecasts, and thereby market outcomes. However, in contrast to REH models' unattainably ambitious precision, an economist can ascertain the consequences of contemplated policy changes only imperfectly as lying within bounds.

Representing a market participant's rational assessment of Knightian uncertainty on the basis of the neo-Muthian hypothesis requires a criterion by which an economist can judge that an empirical approximation adequately resembles his model's ex ante specification. Because developing and applying our approach to building macroeconomic and finance models of outcomes undergoing unforeseeable change exceeds the scope of this paper, we develop such a criterion of adequacy in a companion paper (Frydman and Tabor, 2021). There, we show how invoking the neo-Muthian hypothesis would enable economists to specify two of the main components of macroeconomic and finance models under Knightian uncertainty: the process driving the exogenous variable that undergoes unforeseeable change, and a rational participant's forecast of where this variable's future values might lie.

Frydman et al. (2021) apply the approach introduced here and in Frydman and Tabor (2021) to build a fully-fledged intertemporal finance model of asset prices undergoing unforeseeable change. By acknowledging that an economist and market participants face Knightian uncertainty about asset prices, Frydman et al. (2021) extends Lucas's (1978) model of these prices.

This extension shows that recognizing that an economist faces Knightian 
uncertainty about asset prices enables him to represent the influence of nonfundamental factors, such as market sentiment, on participants' forecasts. In contrast to Barberis et al.'s (1998) presumption that such factors impel market participants to commit systematic errors, our approach can formalize the influence of non-fundamental factors in a consistent model, in the sense of the neo-Muthian hypothesis. Recognizing in a consistent model that participants and an economist face Knightian uncertainty about asset prices reveals that market sentiment plays a key role in aligning market participants' rational decisions more closely with their utility-maximizing objectives.

More broadly, our research suggests that recognizing Knight's seemingly uncontroversial idea that the future is open to change that cannot be specified ex ante with probabilistic rules promises to enhance substantially our understanding of market outcomes and the role of economic policy. 


\section{References}

[1] Barberis, Nicholas C., Shleifer, Andrei and Robert Vishny.(1998), "A Model of Investor Sentiment," Journal of Financial Economics, 49, 307343.

[2] Barberis, Nicholas C. and Richard H. Thaler (2003), "A Survey of Behavioral Finance," in Constantinides, George, Harris, Milton and Rene Stulz (eds.), Handbook of the Economics of Finance, Amsterdam: NorthHolland.

[3] Bordalo, Pedro, Gennaioli, La Porta, Rafael and Andrei Shleifer (2020a), "Expectations of Fundamentals and Stock Market Puzzles," NBER Working Paper No. w27283.

[4] Bordalo, Pedro, Gennaioli, Nicola, Ma, Yueran and Andrei Shleifer (2020b), "Overreaction in Macroeconomic Expectations, "American Economic Review, 110, 2748-2782.

[5] Fama, Eugene F. (1998), "Market Efficiency, Lomg-term Returns, and Behavioral Finance, Journal of Financial Economics, 283-306.

[6] Fiedler, Klaus and Momme von Sydow (2015), "Heuristics and Biases: Beyond Tversky and Kahneman's (1974) Judgment under Uncertainty," in Cognitive Psychology: Revisiting the Classic Studies, Grome, David and Michael W. Eysenck (eds.), Sage Publications, 146-161.

[7] Frydman, Roman and Michael D. Goldberg (2007), Imperfect Knowledge Economics: Exchange Rates and Risk, Princeton University Press.

[8] Frydman, Roman and Edmund S. Phelps (2013), "Which Way Forward for Macroeconomics and Policy Analysis?," in Frydman, Roman and Edmund S. Phelps (eds.), Rethinking Expectations; The Way Forward for Macroeconomics, Princeton University Press, 1-48.

[9] Frydman, Roman and Michael D. Goldberg (2015), "A New Rational Expectations Hypothesis: What Can Economists Really Know About the Future?," A Note Presented at the Institute for New Economic Thinking Plenary Conference, Paris, April 8-11.

[10] Frydman, Roman and Joshua R. Stillwagon (2018), "Fundamental Factors and Extrapolation in Stock-Market Expectations: The Central Role 
of Structural Change," The Journal of Economic Behavior and Organization, 148, 189-198.

[11] Frydman, Roman and Morten Nyboe Tabor (2021), "Applying Kahneman and Tversky's Findings to Modeling Rational Assessments of Knightian Uncertainty," in preparation.

[12] Frydman, Roman, Nicholas Mangee and Joshua R. Stillwagon (2020), "How Market Sentiment Drives Forecasts of Stock Returns," Journal of Behavioral Finance, June.

[13] Frydman, Roman, Johansen, Søren, Rahbek, Anders and Morten Nyboe Tabor (2021), "Acknowledging Knightian Uncertainty in a Model of Asset Prices," in preparation.

[14] Gati, Itamar and Amos Tversky (1982), "Representations of Qualitative and Quantitative Dimensions, " Journal of Experimental Psychology: Human Perception and Performance, 325-340.

[15] Gennaioli, Nicola and Andrei Shleifer (2018), The Crisis of Beliefs: Investor Psychology and Financial Fragility, Princeton University Press.

[16] Hamilton, James D. (2008), "Regime Switching Models," in Steven Durlauf and Lawrence Blume (eds.) New Palgrave Dictionary of Economics, 2nd edition, 53-57, Palgrave McMillan Ltd.

[17] Hansen, Lars Peter (2013), "Uncertainty Outside and Inside Economic Models," The Nobel Prize Lecture, The Nobel Foundation.

[18] Interpretations of Probability (2019), in Stanford Encyclopedia of Philosophy.

[19] Kahneman, Daniel and Amos Tversky (1982), "Subjective Probability: A Judgment of Representativeness," in Daniel Kahneman et al (eds.), Heuristics and Biases, Cambridge University Press.

[20] Knight, Frank H. (1921), Risk, Uncertainty and Profit, Houghton Mifflin.

[21] Lucas, Robert E. Jr. (1976), "Econometric Policy Evaluation: A Critique," In Brunner, Karl and Allan Meltzer (eds.), The Phillips Curve and Labor Markets, Carnegie-Rochester Conference Series on Public Policy. American Elsevier, 19-46.

[22] Lucas, Robert E. Jr. (1978), "Asset Prices in an Exchange Economy," 
Econometrica, 46, 1429-1445.

[23] Lucas, Robert E. Jr. (1995), "The Monetary Neutrality," The Nobel Prize Lecture, The Nobel Foundation.

[24] Lucas, Robert E. Jr. (2005), "Robert E. Lucas, Jr.," in William Breit and Barry T. Hirsch (eds.), Lives of the Laureates: Eighteen Nobel Economists, The MIT Press, 273-297.

[25] Muth, John F. (1961), "Rational Expectations and the Theory of Price Movements," Econometrica, 29, 315-335.

[26] Perron, Pierre (2009), "Econometrics of Structural Change," in Macroeconometrics and Time Series Analysis, Durlauf Steven and L. Blume (eds.), Springer-Verlag, 288-302.

[27] Shiller, Robert J. (1981), "Do Stock Prices Move too Much to be Justified by Subsequent Changes in Dividends," American Economic Review, 71, 421-36.

[28] Simon, Herbert A. (1955), "A Behavioral Model of Rational Choice," The Quarterly Journal of Economics, 69, 99-118.

[29] Thaler, Richard H. (2017), "From Cashews to Nudges: The Evolution of Behavioral Economics," The Nobel Prize Lecture, The Nobel Foundation.

[30] Tversky Amos (1977), "Features of Similarity," Psychological Review, 327352.

[31] Tversky, Amos and Daniel Kahneman (1974), "Judgment under Uncertainty: Heuristics and Biases,". Science, 185, 1124-1130.

[32] Tversky, Amos and Daniel Kahneman (1981), "The Framing of Decisions and the Psychology of Choice," Science, 453-458.

[33] Tversky, Amos and Daniel Kahneman (1983), "Extensional versus Intuitive Reasoning: The Conjunction Fallacy in Probability Judgment," Psychological Review, 90, 293-315.

[34] Von Mises, Richard (1957), Probability, Statistics, and Truth, Dover. 


\section{Appendix: Proofs of Propositions and Corollaries}

\section{Proof of Proposition 3}

Using the identity, in (4), we rewrite $R\left(T \mid H^{p}, H^{n p}\right)=\frac{f\left(T \mid H^{p}\right)}{f\left(T \mid H^{n p}\right)}$ as

$$
R\left(T \mid H^{p}, H^{n p}\right)=\frac{f\left(T \cap F \mid H^{p}\right)+f\left(T \cap F^{C} \mid H^{p}\right)}{f\left(T \cap F \mid H^{n p}\right)+f\left(T \cap F^{C} \mid H^{n p}\right)} .
$$

Hence

$$
R\left(T \cap F \mid H^{p}, H^{n p}\right)>R\left(T \mid H^{p}, H^{n p}\right)
$$

if and only if

$$
\begin{aligned}
& R\left(T \cap F \mid H^{p}, H^{n p}\right) f\left(T \cap F \mid H^{n p}\right)+R\left(T \cap F \mid H^{p}, H^{n p}\right) f\left(T \cap F^{C} \mid H^{n p}\right) \\
> & f\left(T \cap F \mid H^{p}\right)+f\left(T \cap F^{C} \mid H^{p}\right) .
\end{aligned}
$$

From (2) $f\left(T \cap F \mid H^{p}\right)=R\left(T \cap F \mid H^{p}, H^{n p}\right) f\left(T \cap F \mid H^{n p}\right)$. Thus (26) holds if and only if

$$
R\left(T \cap F \mid H^{p}, H^{n p}\right)>R\left(T \cap F^{C} \mid H^{p}, H^{n p}\right) .
$$

\section{Proof of Corollary 4}

Using (4) and $R\left(T \mid H^{p}, H^{n p}\right)=\frac{f\left(T \mid H^{p}\right)}{f\left(T \mid H^{n p}\right)}$, we rewrite

$\Delta(T \cap F, T))=R\left(T \cap F \mid H^{p}, H^{n p}\right)-R\left(T \mid H^{p}, H^{n p}\right)$ as

$$
\Delta(T \cap F, T))=R\left(T \cap F \mid H^{p}, H^{n p}\right)-\frac{f\left(T \cap F \mid H^{p}\right)+f\left(T \cap F^{C} \mid H^{p}\right)}{f\left(T \cap F \mid H^{n p}\right)+f\left(T \cap F^{C} \mid H^{n p}\right)},
$$

which, using $R\left(T \cap F \mid H^{p}, H^{n p}\right)=\frac{f\left(T \cap F \mid H^{p}\right)}{f\left(T \cap F \mid H^{n p}\right)}$, implies that

$\Delta(T \cap F, T)) f\left(T \mid H^{n p}\right)=\frac{R\left(T \cap F \mid H^{p}, H^{n p}\right)\left[f\left(T \cap F^{C} \mid H^{n p}\right)-f\left(T \cap F^{C} \mid H^{p}\right)\right]}{f\left(T \mid H^{n p}\right)}$.

Using (8) and letting $\lambda=\frac{f\left(T \cap F \mid H^{n p}\right)}{f\left(T \mid H^{n p}\right)}<1$ in (28) shows that

$$
R\left(T \cap F \mid H^{p}, H^{n p}\right)-R\left(T \mid H^{p}, H^{n p}\right)=\lambda\left(R\left(T \cap F \mid H^{p}, H^{n p}\right)-R\left(T \cap F^{C} \mid H^{p}, H^{n p}\right)\right) .
$$




\section{Proof of Proposition 10}

Restating (22):

$$
f\left(H^{p} \mid T \cap F\right)>f\left(H^{p} \mid T \cap F^{C}\right)=\frac{n\left(H^{p} \cap T \cap F^{C}\right)}{n\left(T \cap F^{C}\right)} .
$$

Using the identity $n\left(H^{p} \cap T \cap F^{C}\right)=n\left(H^{p} \cap T\right)-n\left(H^{p} \cap T \cap F\right)$, we rewrite the right hand side of (29) as

$$
\frac{n\left(H^{p} \cap T \cap F^{C}\right)}{n\left(T \cap F^{C}\right)}=f\left(H^{p} \mid T\right) \frac{n(T)}{n\left(T \cap F^{C}\right)}-f\left(H^{p} \mid T \cap F\right) \frac{n(T \cap F)}{n\left(T \cap F^{C}\right)} .
$$

Given that $n(T)=n(T \cap F)+n\left(T \cap F^{C}\right)$, using (30) in (29) implies that

$$
f\left(H^{p} \mid T \cap F\right)>f\left(H^{p} \mid T\right) .
$$

